\title{
How cells switch HIPK2 on and off
}

\author{
D Sombroek ${ }^{1}$ and TG Hofmann ${ }^{*, 1}$
}

Homeodomain-interacting protein kinase 2 (HIPK2) is an emerging regulator of cell growth and apoptosis in various cell types, tissues and organisms. Previous work indicates that HIPK2 is a potential tumour suppressor and DNA damage-responsive kinase, which phosphorylation-dependently activates the apoptotic programme by engaging diverse downstream targets, including tumour suppressor p53 and the anti-apoptotic transcriptional corepressor C-terminal binding protein. The regulation of HIPK2, however, remained largely obscure. Recent studies show that HIPK2 activity is mainly controlled at the posttranscriptional level through targeted proteolysis. Caspase-dependent processing triggers HIPK2 hyperactivation, whereas the ubiquitin-proteasome system (UPS) keeps HIPK2 in check by targeting it for degradation. Both HIPK2 hyperactivation and HIPK2 degradation are under the control of transcription factor p53. Negative regulation of HIPK2 by the UPS is abolished in response to DNA damage, which facilitates HIPK2 stabilization and activation. Here we discuss these findings in the context of DNA damage signalling and tumour suppression.

Cell Death and Differentiation (2009) 16, 187-194; doi:10.1038/cdd.2008.154; published online 31 October 2008

DNA damage, if remains unrepaired or damaged cells fail to be eliminated through apoptosis or cellular senescence, poses a serious threat to the organism by causing genomic instability, a main driving force for tumorigenesis. Higher organisms have evolved highly efficient mechanisms, summarized as the DNA damage response (DDR), to cope with genotoxic insult and to fight genomic instability and cancer development. ${ }^{1-3}$ Depending on the cell type and the extent of DNA damage, the DDR provokes different cell fate decisions: cell cycle checkpoint activation, DNA repair and subsequent cellular recovery from damage, or cellular senescence and apoptosis. Recent findings strongly support the concept that the DDR provides a potent innate barrier against cellular transformation and tumorigenesis. In this context, it has been shown that the DDR is not exclusively activated in response to genotoxic stress but also upon oncogenic transformation, and that it is constitutively active in pre-cancerous lesions of humans and mice and drives cells into senescence. ${ }^{4-6}$ These findings further spotlight the DDR as a key tumour-suppressive force. A detailed understanding of the players and the regulatory processes involved in controlling DNA damageinduced cell fate decisions might enable us to manipulate the DDR towards induction of cellular senescence and/or apoptosis, which is a promising approach for improving the efficacy of cancer therapies.

Although the molecular mechanisms underlying cellular decision making and effector pathway shaping upon DNA damage are yet incompletely understood, a number of molecular players have been identified that play an important role in this process. One promising candidate is the serine/ threonine kinase homeodomain-interacting protein kinase 2 (HIPK2). In this review, we highlight the recent findings on HIPK2 function and regulation, with a focus on its role in the cellular response to DNA damage.

\section{DNA Damage Signaling}

Genome integrity is constantly threatened by environmental and cell-derived genotoxic stressors, such as ultraviolet (UV) light, ionizing radiation (IR), alkylating agents and reactive oxygen species (ROS). These genotoxins can induce different types of DNA lesions, including DNA double-strand breaks (DSBs), single-strand breaks, base adducts and intraand inter-strand crosslinks or stalling of replication forks, stimuli that all activate the DDR. ${ }^{3}$

The core principles and the machinery of the DDR seem to be highly conserved during evolution. The ignition of the DDR is controlled by sensor protein complexes, which scan the genomic landscape and sense DNA lesions. DSBs are recognized through the Mre11/Rad50/NBS1 (MRN) complex, which facilitates activation of the DNA damage checkpoint kinase ataxia-telangiectasia mutated (ATM). ${ }^{7}$ In contrast, in response to replicative stress, as caused by stalled or collapsed replication forks during the S-phase of the cell cycle, the checkpoint kinase ATM- and RAD3-related (ATR) gets primarily activated. ${ }^{8}$ To this end, a protein complex

\footnotetext{
${ }^{1}$ Cellular Senescence Group, German Cancer Research Center (DKFZ), DKFZ-ZMBH Alliance, Heidelberg, Germany

${ }^{*}$ Corresponding author: TG Hofmann, Cellular Senescence Group, Deutsches Krebsforschungszentrum (DKFZ), Im Neuenheimer Feld 242, Heidelberg 69120, Germany. Tel: + 496221424 631; Fax: + 496221424 902; E-mail: t.hofmann@dkfz.de

Keywords: DNA damage; HIPK2; ATM; apoptosis; Siah-1; p53

Abbreviations: ATM, ataxia-telangiectasia mutated; ATR, ATM- and RAD3-related; BMP, bone morphogenic protein; CBP, Creb-binding protein; CtBP, C-terminal binding protein; DDR, DNA damage response; HIPK2, homeodomain-interacting protein kinase 2; HMGA1, high-mobility group A1; IR, ionizing irradiation; MDM2, murine double minute 2; NB, nuclear body; PML, promyelocytic leukemia; Shh, sonic hedgehog; Siah-1, seven in absentia homolog-1; TGF- $\beta$, transforming growth factor- $\beta$; UPS, ubiquitin-proteasome system; UV, ultraviolet; WSB-1, WD40 domain and suppressor of cytokine signaling (SOCS) box protein-1

Received 23.6.08; revised 15.9.08; accepted 16.9.08; Edited by JC Marine; published online 31.10.08
} 
consisting of ATR and the ATR-interacting protein (ATRIP) is recruited to the lesion and mediates ATR activation in a TopBP1regulated fashion. ${ }^{9-11}$ Active ATM and ATR induce activation of downstream checkpoint kinases, including Chk1 and Chk2, and this kinase network coordinates the DDR by phosphorylationdependent control of critical downstream regulatory proteins to establish DNA repair, apoptosis or cellular senescence. ${ }^{12}$

A major downstream factor of the DDR is the tumour suppressor and transcription factor p53. p53 is often mutated or functionally inactivated in cancer, ${ }^{13}$ and is well established to counteract cancer development by driving cell growth arrest, apoptosis and senescence. ${ }^{14-16}$ In response to DNA damage, p53 is phosphorylated at multiple sites by various DNA damage-activated protein kinases. For instance, ATM and ATR are known to phosphorylate p53 at Ser15, and the Chk1 and Chk2 at Ser20, two phosphorylation events that contribute to p53 stabilization and transcriptional activation after DNA damage. ${ }^{17}$ In addition, HIPK2 was found to be a DNA damage-responsive kinase that gets activated upon UV, ionizing irradiation and chemotherapeutic drug treatment. ${ }^{18-21}$ HIPK2 phosphorylates p53 at Ser46, a phosphorylation mark occurring in response to severe genotoxic stress, which has been linked to apoptosis induction and cellular senescence. ${ }^{22,23}$

\section{Serine/Threonine Kinase HIPK2}

HIPK2 (an schematic presentation is shown in Figure 1) has been identified due to its interaction with the homeodomain transcription factor NK-3 and is part of the closely related family of HIPKs, which comprises four family members. ${ }^{24}$ HIPK2 is a large, predominantly nuclear localized serine/threonine kinase, with a particular localization to nuclear bodies (NBs) that overlap, at least in part, with polycomb protein bodies. ${ }^{25}$ HIPK2 is conserved from flies to man and plays a critical role in the regulation of cell growth, development, morphogenesis, transcription and cell death in multiple organisms and cell types. ${ }^{18,19,26-30}$ First identified as a transcriptional regulator and corepressor for homeodomain transcription factors, HIPK2 was subsequently recognized as a potent growth suppressor and apoptosis activator (for recent reviews see Calzado et al. ${ }^{31}$ and Rinaldo et al. ${ }^{32}$ ). Accordingly, HIPK2 was found to be a DNA damage-responsive kinase that gets activated upon different DNA damage-inducing stimuli, including UV irradiation, IR or treatment with genotoxic chemotherapeutic drugs such as adriamycin and cisplatin. ${ }^{18-21}$

A recent report showed that HIPK2 exerts a tumoursuppressive function in mouse skin. ${ }^{33}$ HIPK2-/- and HIPK2 + I- mice are tumour prone and develop skin tumours with high frequency when challenged by the classical twostage skin carcinogenesis model using DMBA as initiator and TPA as promoter. Remarkably, skin tumour formation both in the HIPK2 + I- and in the - /- background showed a similarly fast progression to carcinomas, which was accompanied by increased cyclin D expression. ${ }^{33}$ Thus, these in vivo data suggest that HIPK2 functions as a haploinsufficient tumour suppressor in the mouse skin.

Concordant with a possible tumour-suppressor function in man, human cancer cells show some tendency to inactivate HIPK2 through different mechanisms (Figure 2). For instance,
HIPK2 is transcriptionally downregulated in thyroid and breast carcinoma specimens. ${ }^{34}$ In addition, HIPK2 was found to be mutated in a very small subset ( 2 out of 130 cases) of acute myeloid leukemia (AML) and myelodyplastic syndrome (MDS) patients, ${ }^{35}$ although these rare mutations do not clearly support a causative role of HIPK2 in the pathogenesis of these tumours. Interestingly, high-mobility group A1 (HMGA1), a proto-oncogene overexpressed in multiple cancer entities, ${ }^{36}$ was shown to inactivate HIPK2 by removing it from the cell nucleus and retaining it in the cytoplasm. ${ }^{37} \mathrm{~A}$ similar mechanism of action was very recently reported for the potentially leukemogenic PEBP $2 \beta$-SMMHC fusion protein, which prevents AML1/RUNX1 activation by targeting HIPK2 to cytoplasmic filaments. ${ }^{38}$

However, very recent reports also indicate frequent amplification of HIPK2 in pilocytic astrocytomas, the most common glia cell-derived brain tumour affecting children and young adults. ${ }^{39,40}$ Concordantly, HIPK2 overexpression in a glioma cell line did result in increased cell growth and not in growth arrest, as reported for multiple other tumour cell systems. ${ }^{18,19,41-45}$ Whether HIPK2 overexpression in pilocytic astrocytoma has an effect on the response rate of these relatively benign tumour entities to therapy is currently unclear. In addition, the molecular mechanism by which HIPK2 increases cell growth still needs to be clarified. Furthermore, future work is required to clarify whether the growth-suppressive versus growth-driving functions of HIPK2 are restricted to certain cell types or tissues.

Nonetheless, in most cell types analysed, HIPK2 activation is associated with the induction of growth suppression and apoptosis, which is mediated through both p53-dependent and p53-independent HIPK2 signalling routes.

\section{The Role of HIPK2 in p53 Regulation}

In response to severe DNA damage, HIPK2 forms a complex with the tumour suppressor p53 and phosphorylates p53 at Ser46, which in turn activates apoptotic p53-target genes such as PUMA, Bax and Noxa. ${ }^{18,19,21,46,47}$ p53 Ser46 has been previously established to play a crucial role in apoptosis induction in response to severe DNA damage. ${ }^{22}$ In mice, HIPK2 can phosphorylate p53 at Ser58, a site homologous to Ser46, to elicit the cell death programme. ${ }^{48}$ To efficiently phosphorylate p53, HIPK2 uses the tumour suppressor protein promyelocytic leukemia (PML) as a cofactor. ${ }^{49} \mathrm{PML}$ recruits $p 53^{50-52}$ along with HIPK2 into PML-NBs, which suggests that HIPK2 phosphorylates p53 at PML-NBs. ${ }^{53,54}$ This model is consistent with the current view that PML-NBs function as subnuclear modification centres that concentrate factors which regulate p53's post-translational modification status and thereby affect p53 activity. ${ }^{55,56}$

HIPK2 also interacts with the acetyltransferase Crebbinding protein (CBP), which colocalizes with HIPK2 and p53 in PML-NBs. CBP mediates p53 lysine 373 and 382 acetylation, which is stimulated through HIPK2-mediated p53 Ser46 phosphorylation. $^{18}$ Interestingly, p53 Lys373/382 acetylation plays a critical role in induction of apoptosis and cellular senescence, and both responses have been linked to PML-NBs. ${ }^{18,50,52}$ Besides PML and its associated NBs, also Axin, ${ }^{42}$ a scaffold protein known from the $\mathrm{Wnt} / \beta$-catenin 


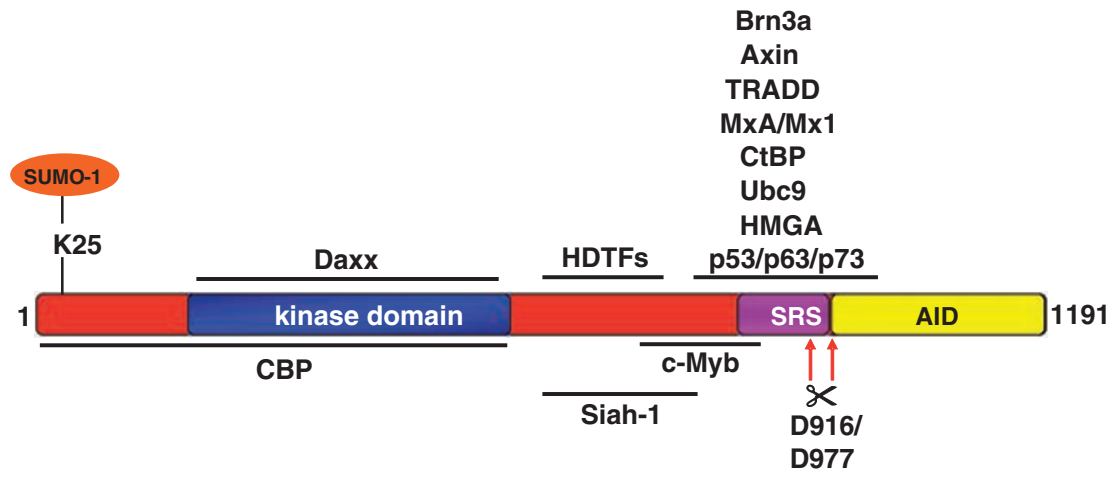

Figure 1 Schematic representation of HIPK2. Functional domains and important residues are shown, along with a selected list of proteins that bind to specific sites (marked with black bars). K25, sumoylation site; HDTFs, homeodomain transcription factor binding; AID, autoinhibitory domain; D916 and D977 are caspase-6 cleavage sites; SRS, speckle-retention signal

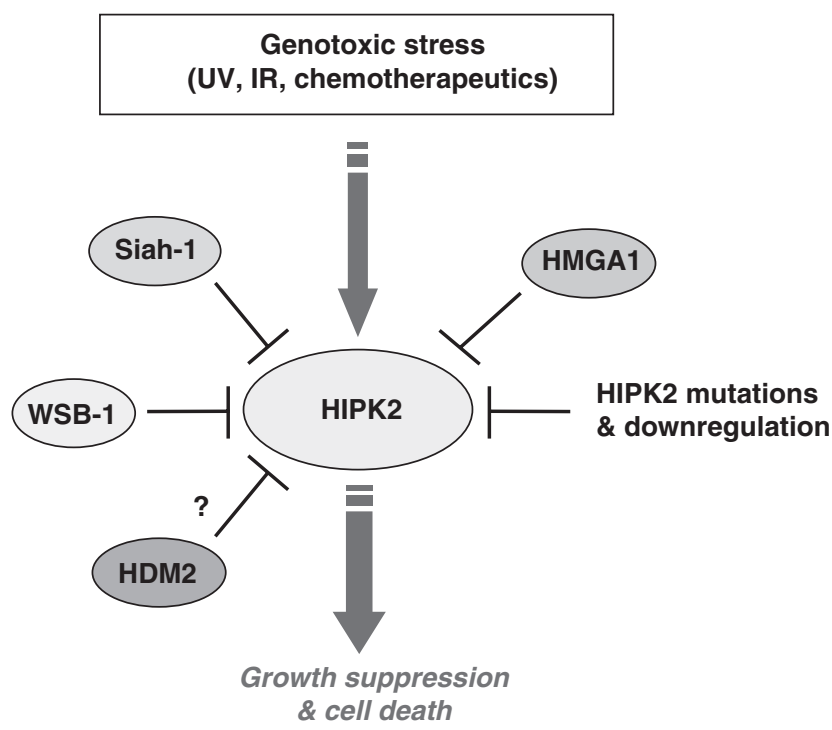

Figure 2 Mechanisms leading to HIPK2 inactivation. HIPK2 is activated in response to genotoxic insult, such as ultraviolet (UV) light, ionizing radiation (IR), or DNA-damaging chemotherapeutics and mediates growth-suppressive and apoptosis-activating signals. HIPK2 activity is restrained in cancer cells through mutations, downregulation of its mRNA or overexpression of HMGA1, which relocalizes HIPK2 into the cytoplasm. The E3 ubiquitin ligases Siah-1 and WSB-1 keep HIPK2 levels low in unstressed cells by facilitating proteasome-dependent HIPK2 degradation. HDM2/MDM2 was proposed to mediate HIPK2 degradation in response to sublethal DNA damage

signalling pathway, the PML-NB components $\mathrm{Sp} 100^{57}$ and $\operatorname{Daxx}^{58}$ and the p53-inducible factor p53DINP1/ TP53INP1 ${ }^{59,60}$ apparently function as cofactors for HIPK2mediated p53 Ser46 phosphorylation by binding both p53 and HIPK2. How these different coactivator functions are coordinated in the cell remains to be studied.

\section{P53-Independent Functions of HIPK2}

HIPK2 can also mediate apoptosis upon DNA damage in the absence of p53. In this context, HIPK2 phosphorylationdependently targets the anti-apoptotic corepressor C-terminal binding protein (CtBP). Phosphorylation of CtBP at Ser422 by
HIPK2 results in proteasomal degradation of CtBP. ${ }^{29}$ In addition, in p53-deficient hepatoma cells HIPK2 is critical for transforming growth factor- $\beta$ (TGF- $\beta$ )-induced apoptosis through activating the JNK signalling pathway. ${ }^{61}$ Remarkably, JNK was also found to mediate CtBP Ser422 phosphorylation and apoptosis induction upon UV damage, suggesting that HIPK2 facilitates CtBP phosphorylation directly as well as indirectly through an engagement of JNK. ${ }^{61,62}$ Furthermore, in neurons HIPK2 was shown to mediate cell death in a Baxdependent manner in response to neurotrophin deprivation. ${ }^{44}$ Interestingly, HIPK2 knock-out mice were found to suffer from a severe neurodegenerative disorder, which was characterized by a peri- and postnatal apoptotic loss of dopaminergic neurons in the substantia nigra, thus resembling a Parkinson's disease-like phenotype. ${ }^{30}$ These data indicate that in dopaminergic neurons HIPK2 obviously acts as a survival factor rather than an apoptosis activator.

Besides its function in apoptosis regulation, HIPK2 is also critical for signal transduction pathways induced by extracellular ligands or morphogens, such as Wnt, bone morphogenic protein (BMP) and TGF- $\beta$, and transcriptional regulation. ${ }^{31,32}$ In line with its reported corepressor function, HIPK2 was found to mediate DNA damage-induced gene repression through interaction with the E3 SUMO ligase polycomb protein 2 (Pc2), ${ }^{25}$ a component of the polycomb repressor complex 2. HIPK2 has been previously shown to be covalently modified by SUMO-1, which does not affect its p53activating function, but its JNK-activating function. ${ }^{63,64}$ Once activated after DNA damage, HIPK2 may phosphorylate Pc2 and thereby increase its ligase activity, which in turn leads to an increased SUMO modification of HIPK2 and an enhanced corepressor activity, thus suggesting a mechanism for gene repression in response to genotoxic stress. ${ }^{25}$ However, how this mechanism functionally contributes to the DDR remains to be clarified.

\section{HIPK2 Regulation by the Ubiquitin Ligases Siah-1 and WSB-1}

Several reports noted an increase in HIPK2 protein levels in response to DNA-damaging treatments, including IR, UV or cisplatin, ${ }^{19-21}$ but until recently the molecular basis for this 


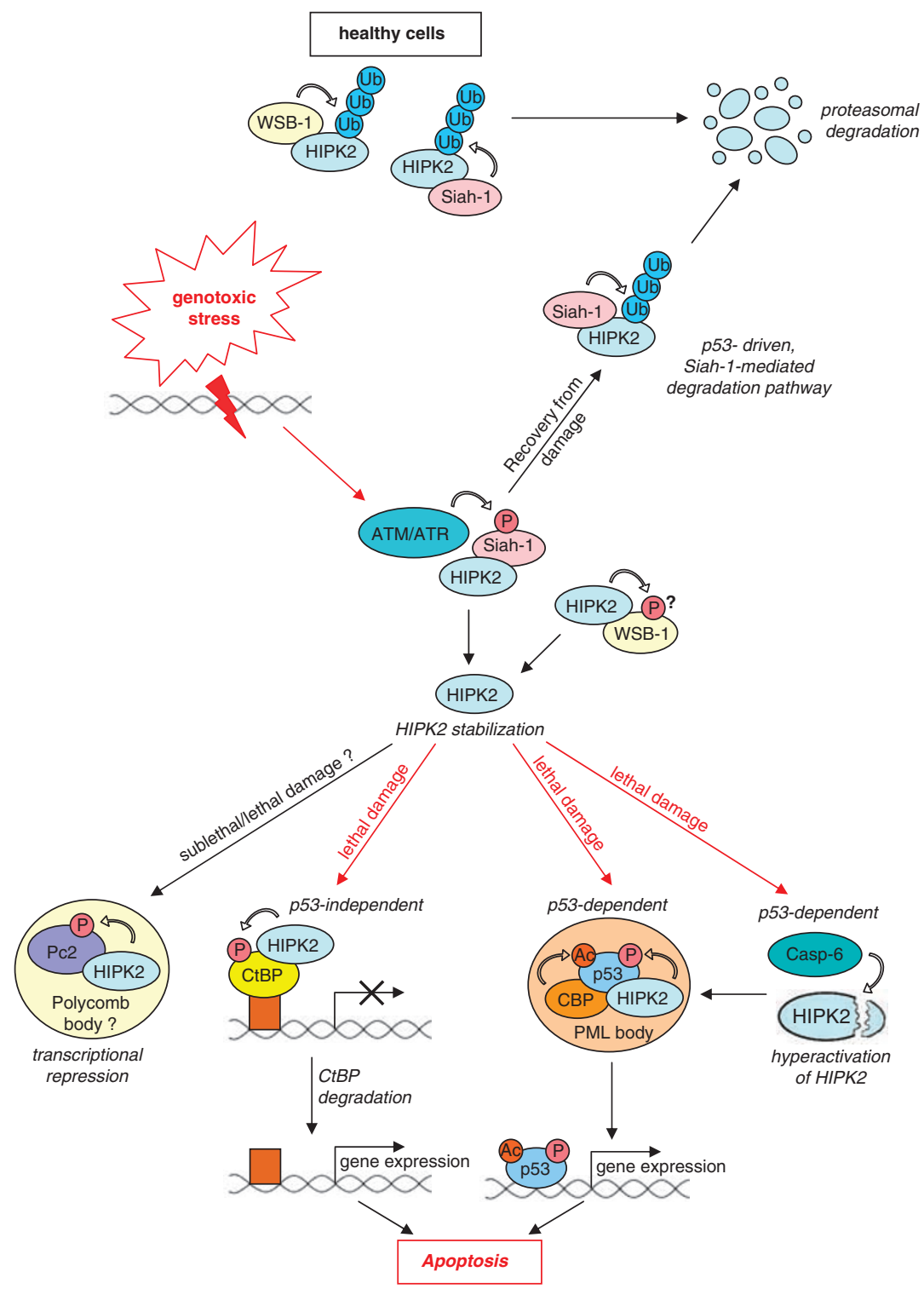

Figure 3 HIPK2 regulation and effector functions upon DNA damage. Unstressed cells constantly degrade HIPK2 through complex formation with the ubiquitin ligases Siah-1 and WSB-1, which directly ubiquitinate HIPK2 and thereby mark it for proteasome-dependent degradation. Genotoxic stress triggers activation of the checkpoint kinases ATM and ATR which phosphorylate Siah-1 and thereby trigger dissociation of Siah-1 from HIPK2, resulting in HIPK2 stabilization and activation. Active HIPK2 might phosphorylate WSB-1, which leads to the disruption of the HIPK2-WSB-1 complex. In response to severe DNA damage, HIPK2 activates the apoptotic machinery through complex formation and direct phosphorylation of p53 and CtBP. In addition, caspase-dependent cleavage of HIPK2 results in a hyperactive kinase and further potentiates its apoptotic functions. HIPK2 also phosphorylates the E3 SUMO ligase Pc2, which results in transcriptional repression of Pc2 target genes. During the recovery from sublethal DNA damage, HIPK2 is again downregulated through a p53-driven, Siah-1-dependent degradation pathway, to prevent unscheduled cell death activation

HIPK2 accumulation remained unexplained. Recent evidence indicates that HIPK2 is an unstable protein with a high turnover in unstressed cells due to degradation by the ubiquitin-proteasome system (UPS). ${ }^{65,66}$

Three E3 ubiquitin ligases have been recently reported to regulate HIPK2 steady-state expression levels: the WDrepeat ligase WSB-1 (WD-repeat and suppressor of cytokine signaling (SOCS) box-containing-1) and the RING family ligases Siah-1 (seven in absentia homolog-1) and Siah-2. ${ }^{65,66}$ Siah-1 associates with HIPK2 through its C-terminal region, which contains its substrate-binding domain, and directly mediates HIPK2 poly-ubiquitination. ${ }^{66}$ In addition, HIPK2 and
Siah-1 colocalize under native and overexpression conditions in NBs, suggesting that HIPK2 ubiquitination may take place at NBs. Similarly, WSB-1 also forms a complex with HIPK2 and is capable of triggering HIPK2 ubiquitination and degradation. $^{42}$ Whether the HIPK2-WSB-1 complex is associated with a distinct subcellular localization has not been addressed yet.

Reduction of endogenous WSB-1, Siah-1 or Siah-2 expression by RNA interference was reported to increase the half-life of HIPK2, thus resulting in HIPK2 accumulation. ${ }^{65,66}$ In line with the cell death-regulating activities of HIPK2, both WSB-1 and Siah-1 knockdown markedly 
H.sapiens Siah-1a

H.sapiens Siah-1b

M.musculus Siah-1a

M.musculus Siah-1b

X.laevis Siah-1

D.rerio

Siah-1

\begin{abstract}
12-TSKCPPSQRVPALTGT
43-TSKCPPSQRVPALTGT

12-TSKCPPSQRVPALTGT

12-TSKCPPSQRVPALTDT

12-TSKCPPSQRVPALTGT

16-TSKCPPSQRVPTLSGT
\end{abstract}

Figure 4 Evolutionary conservation of the ATM/ATR phosphorylation site in Siah-1 proteins. Siah proteins of the indicated species were aligned to identify potential ATM and ATR consensus phosphorylation SQ/TQ sites. The accession numbers for the proteins indicated are the following: Homo sapiens Siah-1a (NP_003022); H. sapiens Siah-1b (NP_001006611); Mus musculus Siah-1a (NP_33198); M. musculus Siah-1b (NP_0033199); Xenopus laevis (NP_001085438); Danio rerio (NP_955815). The conserved SQ motif is shaded in grey colour

sensitized cells for DNA damage-induced apoptosis as activated by adriamycin treatment or UV irradiation. ${ }^{65,66}$ These data indicate a critical role for WSB-1 and Siah-1 in controlling HIPK2 turnover in proliferating cells and in the control of radiation- and chemosensitivity.

The expression of WSB-1 is induced by the morphogen sonic hedgehog (Shh), which plays an important role during development. ${ }^{67}$ Recently, also HIPK2 has been linked to the hedgehog signalling pathway and development, suggesting that WSB-1 could act as a direct link between HIPK2 and Shh signalling. How HIPK2 regulates development and its precise function in the hedgehog pathway remain to be elucidated.

\section{Two Faces of p53 in HIPK2 Regulation: Caspase- Dependent Activation Versus Proteasome-Dependent Degradation}

p53 is a major mediator for HIPK2's apoptotic function by way of serving as a direct substrate. Furthermore, HIPK2 activity is tightly regulated in a p53-dependent fashion. Remarkably, p53 appears to coordinate both HIPK2 activation and inactivation (see the overview in Figure 3).

For example, in response to lethal adriamycin treatment, HIPK2 has been shown to be processed at aspartate residues 916 and 977 in a caspase-dependent manner. ${ }^{31,68}$ Caspasedependent cleavage removes an inhibitory $\mathrm{C}$-terminal domain from HIPK2 (see Figure 1), resulting in a hyperactive kinase, which potentiates p53 Ser46 phosphorylation and activation of the cell death machinery. Interestingly, HIPK2 processing was found to require caspase-6, which is an established p53target gene. ${ }^{69}$ Thus, p53 can drive a feed-forward loop to potentiate HIPK2's apoptotic activity through its caspasedependent processing in response to severe genotoxic stress.

In addition, p53 was also shown to negatively regulate HIPK2 function in response to sublethal DNA damage. ${ }^{66,70}$ Consistently, p53-deficient cells were found to express elevated HIPK2 levels, and artificial expression of p53 can drive proteasome-dependent HIPK2 degradation. ${ }^{66,70}$ In line with these observations, HIPK2 is degraded in a p53- and proteasome-dependent fashion during the recovery phase from mild DNA damage. ${ }^{66}$ One report proposed that the p53inducible E3 ubiquitin ligase MDM2 (murine double minute 2)/ HDM2 acts as a p53-induced negative regulator of HIPK2 driving HIPK2 ubiquitination and degradation during mild DNA damage. ${ }^{70}$ MDM2 and its human homolog HDM2 were shown to interact with HIPK2 upon DNA damage and proposed to mediate HIPK2 poly-ubiquitination and degradation during sublethal DNA damage. ${ }^{70}$ In a recent study, we failed to confirm a role for MDM2/HDM2 in p53-driven HIPK2 degradation. ${ }^{66}$ In contrast to the findings reported by Rinaldo and collegues, no requirement of HDM2/MDM2 in p53-dependent HIPK2 degradation could be confirmed by using MDM2-deficient mouse embryonic fibroblasts as well as human cells depleted of HDM2 by RNAi. These discrepancies remain to be solved in the future. Depletion of the p53-inducible E3 ligase Siah-1, ${ }^{71-74}$ however, resulted in a clear inhibition of HIPK2 degradation, both upon sublethal DNA damage and upon ectopic expression of p53, indicating a critical role of Siah-1 in p53-driven HIPK2 degradation. ${ }^{66}$ Whether WSB-1 also plays a role in the p53-induced HIPK2 degradation is currently not known. In summary, there is solid evidence that $\mathrm{p} 53$, besides its role as a mediator in the HIPK2-cell death axis, can both potentiate and terminate HIPK2 activity by controlling HIPK2 proteolysis.

\section{HIPK2 Regulation by the DNA Damage Checkpoint Kinases ATM and ATR}

DNA damage triggers HIPK2 stabilization and accumulation, although p53 - which drives proteasome-dependent HIPK2 degradation - is potently activated. ${ }^{66}$ These findings argue for a cellular mechanism by which HIPK2 gets uncoupled from UPS-dependent degradation in response to DNA damage. In line with this assumption, HIPK2 stabilization upon DNA damage requires the DNA damage checkpoint kinases $\mathrm{ATM}^{21,66}$ and $\mathrm{ATR}^{66}$ (Figure 3). Pharmacological inhibition or RNAi-mediated depletion of ATM and ATR prevents HIPK2 accumulation and stabilization after DNA damage, indicating that HIPK2 acts downstream of ATM and ATR in the DDR. ${ }^{21,66}$

Hence, what is the mechanism of ATM and ATR in HIPK2 stabilization? Both ATM and ATR expression efficiently trigger disruption of the HIPK2-Siah-1 complex and this effect is dependent on the kinase activity of ATM/ATR. ${ }^{66}$ Concordantly, both ATM and ATR are capable of phosphorylating human Siah-1 at Ser19, which is part of an ATM/ATR consensus phosphorylation site. Ser19 is critical for ATM/ ATR-mediated disruption of the HIPK2-Siah-1 complex, although mutation of Ser19 to Ala did not fully prevent complex disruption, suggesting that additional mechanisms are involved in breaking the HIPK2-Siah-1 interaction in response to DNA damage.${ }^{66}$ Consistent with an important role of ATM and ATR in regulating the human HIPK2-Siah-1 complex, the consensus phosphorylation motif is conserved in Siah-1 proteins of mice, frogs and fish (Figure 4). In contrast, human Siah-2 does not contain any ATM/ATR consensus phosphorylation sites (not shown), suggesting no direct regulation through ATM and ATR.

Remarkably, also the HIPK2-WSB-1 complex is dissolved in response to DNA damage. ${ }^{65}$ The kinase function of HIPK2 seems to play a critical role for the dissociation of HIPK2 from WSB-1, as a kinase-deficient HIPK2 mutant failed to dissociate from WSB-1 upon adriamycin treatment. Although these results strongly suggest that WSB-1 might be a direct 
substrate protein for HIPK2, it remains to be shown whether HIPK2 indeed controls its interaction with WSB-1 through direct phosphorylation (Figure 3).

\section{HIPK2 and Siah in Transformation and Tumorigenesis}

The Ras signalling pathway is a critical mediator of cell proliferation in response to growth factor receptor activation. Hyperactive Ras point mutants, such as H-RasV12, show an oncogenic potential and are found in human cancer specimens of the colon, pancreas, lung and skin. ${ }^{75}$ Interestingly, Siah proteins play also a critical role in Ras signaling. The function of Siah proteins in the Ras pathway is evolutionarily conserved between flies and man, and human Siah-1 and Siah-2 were recently shown to be essential for RasV12mediated transformation and tumorigenesis. ${ }^{76,77}$ Furthermore, the catalytic activity of Siah proteins seems to be strictly required for their role in transformation and tumourigenesis, ${ }^{75}$ implying that Siah facilitates ubiquitination and subsequent proteasomal degradation of specific cellular factors that counteract Ras-driven transformation. Identification of these substrates will warrant important insight into the mechanism of Ras-driven tumourigenesis.

HIPK2 knock-out mice rapidly develop carcinomas in situ and invasive squamous cell carcinomas of the skin in the twostage skin carcinogenesis model. ${ }^{33}$ Analysis of malignant skin lesions in HIPK2-compromised mice confirmed the presence of the typical activating $\mathrm{H}-\mathrm{RasV} 12$ mutation, which is a commonly observed mutation in this mouse tumour model. Furthermore, transfection of H-RasV12 in HIPK2-I- cells resulted in increased colony growth, indicating that HIPK2 expression represents a barrier against oncogenic transformation. ${ }^{33}$ Collectively, these results suggest that HIPK2 is a critical target on the road to cell transformation and tumour development.

Siah ligases are also important players in the hypoxia pathway, a highly tumour-relevant pathway, which induces angiogenesis in order to facilitate tumour outgrowth. ${ }^{78}$ The transcription factor hypoxia-inducible factor $-1 \alpha$ (HIF- $1 \alpha$ ) is a master regulator of the hypoxic response. HIF- $1 \alpha$ levels are kept low through the UPS, which is facilitated by hydroxylation of HIF-1 $\alpha$ through the prolyl-hydroxylases PHD1, PHD2 and PHD3. ${ }^{79}$ Under hypoxic conditions, Siah-1 and Siah-2 target PHD1 and PHD3 for ubiquitination and subsequent proteasomal destruction, thus allowing HIF- $1 \alpha$ stabilization and the induction of its target genes like VEGF. ${ }^{80}$ Interestingly, a link between HIPK2 and vasculogenesis and angiogenesis has been reported recently. HIPK1/HIPK2 double knock-out mouse embryos, in contrast to HIPK1-/- embryos, show severe defects in vessel formation, including vasculogenesis and angiogenesis, indicating a critical role of HIPK2 in the development of vascular endothelial cells. ${ }^{81}$ The intriguing question whether HIPK2 is also a target for Siah-dependent degradation during hypoxia remains to be answered.

\section{Concluding Remarks and Future Perspectives}

There is strong evidence that HIPK2 serves as a central regulator of life-and-death decisions from flies to man. ${ }^{18,19,26}$
HIPK2 is functionally connected to a broad range of cellular signalling pathways with important functions in embryonic development, morphogenesis and cell fate control (Wnt, TGF- $\beta$, BMP, Shh, DNA damage), which underscores a critical role for HIPK2 in integrating cellular signals in order to define and orchestrate the subsequent cellular response.

Currently, the best understood function of HIPK2 is its role in DNA damage signalling. Despite this fact, a number of important questions concerning the function of HIPK2 in this pathway remain to be answered. Although HIPK2 plays a fundamental role in apoptosis induction upon DNA damage by phosphorylating p53 at Ser46 and CtBP at Ser422, it is currently unclear whether there are further HIPK2 substrates critical for apoptosis induction by HIPK2. In addition, HIPK2 is also stabilized in response to sublethal DNA damage, when p53 Ser46 phosphorylation, CtBP degradation and apoptosis induction are absent. This strongly argues for an additional, non-apoptotic role of HIPK2 in response to DNA damage. However, both the substrates phosphorylated by HIPK2 and its exact function in this setting remain to be defined. In order to discriminate between the apoptotic and non-apoptotic functions of HIPK2, studies using clearly defined conditions of sublethal and lethal DNA damage are of fundamental importance.

Furthermore, important questions relating to the HIPK2-Siah1 and HIPK2-WSB-1 link remain to be solved in the future. Recent reports established that the DDR provides an efficient anti-tumour barrier in man. Therefore, it will be interesting to analyse a function of Siah-1 and WSB-1 in tumorigenesis and the effect of a functional/dysfunctional DDR in this setting. Finally, given the important role of Siah proteins in the hypoxic response, a main pathway for the establishment of tumour growth and metastasis, it will be interesting to determine whether HIPK2 may play also a role in hypoxia.

Acknowledgements. We are grateful for the support by the Deutsche Forschungsgemeinschaft, the Deutsche Krebshilfe and the Landesstiftung BadenWürttemberg. We apologize to all the colleagues who made important contributions that could not be cited here due to space limitations. We are grateful to Dr. E Krieghoff-Henning for his critically reading of the manuscript and helpful suggestions.

1. Zhou BB, Elledge SJ. The DNA damage response: putting checkpoints in perspective. Nature 2000; 408: 433-439.

2. Rouse J, Jackson SP. Interfaces between the detection, signaling, and repair of DNA damage. Science 2002; 297: 547-551.

3. Harper JW, Elledge SJ. The DNA damage response: ten years after. Mol Cell 2007; 28: 739-745.

4. Bartkova J, Horejsi Z, Koed K, Kramer A, Tort F, Zieger $\mathrm{K}$ et al. DNA damage response as a candidate anti-cancer barrier in early human tumorigenesis. Nature 2005; 434: 864-870.

5. Di Micco R, Fumagalli M, Cicalese A, Piccinin S, Gasparini P, Luise C et al. Oncogeneinduced senescence is a DNA damage response triggered by DNA hyper-replication. Nature 2006; 444: 638-642

6. Bartkova J, Rezaei N, Liontos M, Karakaidos P, Kletsas D, Issaeva N et al. Oncogeneinduced senescence is part of the tumorigenesis barrier imposed by DNA damage checkpoints. Nature 2006; 444: 633-637.

7. Shiloh Y. ATM and related protein kinases: safeguarding genome integrity. Nat Rev Cancer 2003; 3: 155-168.

8. Abraham RT. Cell cycle checkpoint signaling through the ATM and ATR kinases. Genes Dev 2001; 15: 2177-2196.

9. Falck J, Coates J, Jackson SP. Conserved modes of recruitment of ATM, ATR and DNA-PKcs to sites of DNA damage. Nature 2005; 434: 605-611.

10. Kumagai A, Lee J, Yoo HY, Dunphy WG. TopBP1 activates the ATR-ATRIP complex. Cell 2006; 124: 943-955. 
11. Kumagai A, Dunphy WG. How cells activate ATR. Cell Cycle 2006; 5: 1265-1268.

12. Bartek J, Lukas J. DNA damage checkpoints: from initiation to recovery or adaptation. Curr Opin Cell Biol 2007; 19: 238-245.

13. Hollstein M, Sidransky D, Vogelstein B, Harris CC. p53 mutations in human cancers. Science 1991; 253: 49-53.

14. Vogelstein B, Lane D, Levine AJ. Surfing the p53 network. Nature 2000; 408: 307-310.

15. Oren M. Decision making by p53: life, death and cancer. Cell Death Differ 2003; 10: 431-442.

16. Vousden KH, Lu X. Live or let die: the cell's response to p53. Nat Rev Cancer 2002; 2 594-604.

17. Bode AM, Dong Z. Post-translational modification of $p 53$ in tumorigenesis. Nat Rev Cancer 2004; 4: 793-805.

18. Hofmann TG, Moller A, Sirma H, Zentgraf H, Taya $\mathrm{Y}$, Droge $W$ et al. Regulation of $p 53$ activity by its interaction with homeodomain-interacting protein kinase-2. Nat Cell Biol 2002; 4: 1-10.

19. D'Orazi G, Cecchinelli B, Bruno T, Manni I, Higashimoto Y, Saito S et al. Homeodomaininteracting protein kinase-2 phosphorylates p53 at Ser 46 and mediates apoptosis. Nat Cell Biol 2002; 4: 11-19.

20. Di Stefano V, Rinaldo C, Sacchi A, Soddu S, D'Orazi G. Homeodomain-interacting protein kinase-2 activity and p53 phosphorylation are critical events for cisplatin-mediated apoptosis. Exp Cell Res 2004; 293: 311-320.

21. Dauth I, Kruger J, Hofmann TG. Homeodomain-interacting protein kinase 2 is the ionizing radiationactivated p53 serine 46 kinase and is regulated by ATM. Cancer Res 2007; 67: 2274-2279.

22. Oda K, Arakawa H, Tanaka T, Matsuda K, Tanikawa C, Mori T et al. p53AIP1, a potential mediator of p53-dependent apoptosis, and its regulation by Ser-46-phosphorylated p53. Cell 2000; 102: 849-862.

23. Kurihara A, Nagoshi H, Yabuki M, Okuyama R, Obinata M, Ikawa S. Ser46 phosphorylation of p53 is not always sufficient to induce apoptosis: multiple mechanisms of regulation of p53-dependent apoptosis. Genes Cells 2007; 12: 853-861.

24. Kim YH, Choi CY, Lee SJ, Conti MA, Kim Y. Homeodomain-interacting protein kinases, a novel family of co-repressors for homeodomain transcription factors. J Biol Chem 1998; 273: 25875-25879.

25. Roscic A, Moller A, Calzado MA, Renner F, Wimmer VC, Gresko E et al. Phosphorylationdependent control of Pc2 SUMO E3 ligase activity by its substrate protein HIPK2. Mol Cell 2006; 24: 77-89.

26. Link N, Chen P, Lu WJ, Pogue K, Chuong A, Mata M et al. A collective form of cell death requires homeodomain interacting protein kinase. J Cell Biol 2007; 178: 567-574.

27. Isono K, Nemoto K, Li Y, Takada Y, Suzuki R, Katsuki M et al. Overlapping roles for homeodomain-interacting protein kinases hipk1 and hipk2 in the mediation of cell growth in response to morphogenetic and genotoxic signals. Mol Cell Biol 2006; 26: 2758-2771.

28. Wiggins AK, Wei G, Doxakis E, Wong C, Tang AA, Zang K et al. Interaction of Brn3a and HIPK2 mediates transcriptional repression of sensory neuron survival. J Cell Biol 2004; 167: 257-267.

29. Zhang Q, Yoshimatsu Y, Hildebrand J, Frisch SM, Goodman RH. Homeodomain interacting protein kinase 2 promotes apoptosis by downregulating the transcriptional corepressor CtBP. Cell 2003; 115: 177-186.

30. Zhang J, Pho V, Bonasera SJ, Holzmann J, Tang AT, Hellmuth J et al. Essential function of HIPK2 in TGFbeta-dependent survival of midbrain dopamine neurons. Nat Neurosci 2007; 10: $77-86$.

31. Calzado MA, Renner F, Roscic A, Schmitz ML. HIPK2: a versatile switchboard regulating the transcription machinery and cell death. Cell Cycle 2007; 6: 139-143.

32. Rinaldo C, Prodosmo A, Siepi F, Soddu S. HIPK2: a multitalented partner for transcription factors in DNA damage response and development. Biochem Cell Biol 2007; 85: 411-418.

33. Wei G, Ku S, Ma GK, Saito S, Tang AA, Zhang J et al. HIPK2 represses beta-cateninmediated transcription, epidermal stem cell expansion, and skin tumorigenesis. Proc Natl Acad Sci USA 2007; 104: 13040-13045.

34. Pierantoni GM, Bulfone A, Pentimalli F, Fedele M, luliano R, Santoro $M$ et al. The homeodomain-interacting protein kinase 2 gene is expressed late in embryogenesis and preferentially in retina, muscle, and neural tissues. Biochem Biophys Res Commun 2002; 290: 942-947.

35. Li XL, Arai Y, Harada H, Shima Y, Yoshida H, Rokudai S et al. Mutations of the HIPK2 gene in acute myeloid leukemia and myelodysplastic syndrome impair AML1- and p53-mediated transcription. Oncogene 2007; 26: 7231-7239.

36. Fusco A, Fedele M. Roles of HMGA proteins in cancer. Nat Rev Cancer 2007; 7: 899-910

37. Pierantoni GM, Rinaldo C, Mottolese M, Di Benedetto A, Esposito F, Soddu S et al. Highmobility group $A 1$ inhibits p53 by cytoplasmic relocalization of its proapoptotic activator HIPK2. J Clin Invest 2007; 117: 693-702.

38. Wee HJ, Voon DC, Bae SC, Ito Y. PEBP2\{beta\}/CBF\{beta\}-dependent phosphorylation of RUNX1 and p300 by HIPK2: implications for leukemogenesis. Blood 2008, DOI: 10.1182/Blood-2008-01-134122.

39. Deshmukh H, Yeh TH, Yu J, Sharma MK, Perry A, Leonard JR et al. High-resolution, dualplatform aCGH analysis reveals frequent HIPK2 amplification and increased expression in pilocytic astrocytomas. Oncogene 2008; 27: 4745-4751.

40. Bar EE, Lin A, Tihan T, Burger PC, Eberhart CG. Frequent gains at chromosome 7q34 involving BRAF in pilocytic astrocytoma. J Neuropathol Exp Neurol 2008; 67: 878-887.

41. Pierantoni GM, Fedele M, Pentimalli F, Benvenuto G, Pero R, Viglietto G et al. High mobility group I $(Y)$ proteins bind HIPK2, a serine-threonine kinase protein which inhibits cell growth. Oncogene 2001; 20: 6132-6141.
42. Rui Y, Xu Z, Lin S, Li Q, Rui H, Luo W et al. Axin stimulates p53 functions by activation of HIPK2 kinase through multimeric complex formation. EMBO J 2004; 23: 4583-4594.

43. Giraud S, Diaz-Latoud C, Hacot S, Textoris J, Bourette RP, Diaz JJ. US11 of herpes simplex virus type 1 interacts with HIPK2 and antagonizes HIPK2-induced cell growth arrest. J Virol 2004; 78: 2984-2993.

44. Doxakis E, Huang EJ, Davies AM. Homeodomain-interacting protein kinase-2 regulates apoptosis in developing sensory and sympathetic neurons. Curr Biol 2004; 14: 1761-1765.

45. Kim EJ, Park JS, Um SJ. Identification and characterization of HIPK2 interacting with p73 and modulating functions of the p53 family in vivo. J Biol Chem 2002; 29: 29.

46. Mayo LD, Seo YR, Jackson MW, Smith ML, Rivera Guzman J, Korgaonkar CK et al. Phosphorylation of human p53 at serine 46 determines promoter selection and whether apoptosis is attenuated or amplified. J Biol Chem 2005; 280: 25953-25959.

47. Ichwan SJ, Yamada S, Sumrejkanchanakij P, Ibrahim-Auerkari E, Eto K, Ikeda MA. Defect in serine 46 phosphorylation of p53 contributes to acquisition of p53 resistance in oral squamous cell carcinoma cells. Oncogene 2006; 25: 1216-1224.

48. Cecchinelli B, Porrello A, Lazzari C, Gradi A, Bossi G, D'Angelo M et al. Ser58 of mouse p53 is the homologue of human Ser46 and is phosphorylated by HIPK2 in apoptosis. Cell Death Differ 2006; 13: 1994-1997.

49. Moller A, Sirma H, Hofmann TG, Rueffer S, Klimczak E, Droge W et al. PML is required for homeodomain-interacting protein kinase 2 (HIPK2)-mediated p53 phosphorylation and cell cycle arrest but is dispensable for the formation of HIPK domains. Cancer Res 2003; 63: 4310-4314.

50. Guo A, Salomoni P, Luo J, Shih A, Zhong S, Gu W et al. The function of PML in p53dependent apoptosis. Nat Cell Biol 2000; 2: 730-736.

51. Fogal V, Gostissa M, Sandy P, Zacchi P, Sternsdorf T, Jensen K et al. Regulation of p53 activity in nuclear bodies by a specific PML isoform. EMBO J 2000; 19: 6185-6195.

52. Pearson M, Carbone R, Sebastiani C, Cioce M, Fagioli M, Saito S et al. PML regulates p53 acetylation and premature senescence induced by oncogenic Ras. Nature 2000; 406: 207-210.

53. Hofmann TG, Will H. Body language: the function of PML nuclear bodies in apoptosis regulation. Cell Death Differ 2003; 10: 1290-1299.

54. Bernardi R, Pandolfi PP. Role of PML and the PML-nuclear body in the control of programmed cell death. Oncogene 2003; 22: 9048-9057.

55. Krieghoff-Henning E, Hofmann TG. Role of nuclear bodies in apoptosis signalling. Biochim Biophys Acta 2008, doi:10.1016/j.bbamcr.2008.07.002.

56. Bernardi R, Pandolfi PP. Structure, dynamics and functions of promyelocytic leukaemia nuclear bodies. Nat Rev Mol Cell Biol 2007; 8: 1006-1016.

57. Moller A, Sirma H, Hofmann TG, Staege H, Gresko E, Ludi KS et al. Sp100 is important for the stimulatory effect of homeodomain-interacting protein kinase-2 on p53-dependent gene expression. Oncogene 2003; 22: 8731-8737.

58. Li Q, Wang X, Wu X, Rui Y, Liu W, Wang J et al. Daxx cooperates with the Axin/HIPK2/p53 complex to induce cell death. Cancer Res 2007; 67: 66-74.

59. Okamura S, Arakawa H, Tanaka T, Nakanishi H, Ng CC, Taya Y et al. p53DINP1, a p53inducible gene, regulates p53-dependent apoptosis. Mol Cell 2001; 8: 85-94.

60. Tomasini R, Samir AA, Carrier A, Isnardon D, Cecchinelli B, Soddu S et al. TP53INP1s and homeodomain-interacting protein kinase-2 (HIPK2) are partners in regulating p53 activity. J Biol Chem 2003; 278: 37722-37729.

61. Hofmann TG, Stollberg N, Schmitz ML, Will H. HIPK2 regulates transforming growth factorbeta-induced c-Jun $\mathrm{NH}(2)$-terminal kinase activation and apoptosis in human hepatoma cells. Cancer Res 2003; 63: 8271-8277.

62. Wang SY, lordanov M, Zhang Q. C-Jun NH2-terminal kinase promotes apoptosis by downregulating the transcriptional co-repressor CtBP. J Biol Chem 2006; 281: 34810-34815.

63. Hofmann TG, Jaffray E, Stollberg N, Hay RT, Will H. Regulation of homeodomaininteracting protein kinase 2 (HIPK2) effector function through dynamic small ubiquitinrelated modifier-1 (SUMO-1) modification. J Biol Chem 2005; 280: 29224-29232.

64. Gresko E, Moller A, Roscic A, Schmitz ML. Covalent modification of human homeodomain interacting protein kinase 2 by SUMO-1 at lysine 25 affects its stability. Biochem Biophys Res Commun 2005; 329: 1293-1299.

65. Choi DW, Seo YM, Kim EA, Sung KS, Ahn JW, Park SJ et al. Ubiquitination and degradation of homeodomain-interacting protein kinase 2 by WD40 repeat/SOCS box protein WSB-1. J Biol Chem 2008; 283: 4682-4689.

66. Winter M, Sombroek D, Dauth I, Moehlenbrink J, Scheuermann K, Crone J et al. Control of HIPK2 stability by ubiquitin ligase Siah-1 and checkpoint kinases ATM and ATR. Nat Cell Biol 2008; 10: 812-824

67. Vasiliauskas D, Hancock S, Stern CD. SWiP-1: novel SOCS box containing WD-protein regulated by signalling centres and by Shh during development. Mech Dev 1999; 82: 79-94.

68. Gresko E, Roscic A, Ritterhoff S, Vichalkovski A, Del Sal G, Schmitz ML. Autoregulatory control of the p53 response by caspase-mediated processing of HIPK2. EMBO J 2006; 25: 1883-1894.

69. MacLachlan TK, El-Deiry WS. Apoptotic threshold is lowered by p53 transactivation of caspase-6. Proc Natl Acad Sci USA 2002; 99: 9492-9497.

70. Rinaldo C, Prodosmo A, Mancini F, lacovelli S, Sacchi A, Moretti F et al. MDM2-regulated degradation of HIPK2 prevents p53Ser46 phosphorylation and DNA damage-induced apoptosis. Mol Cell 2007; 25: 739-750.

71. Matsuzawa S, Takayama S, Froesch BA, Zapata JM, Reed JC. p53-inducible human homologue of Drosophila seven in absentia (Siah) inhibits cell growth: suppression by BAG-1. EMBO J 1998; 17: 2736-2747. 
72. Matsuzawa SI, Reed JC. Siah-1, SIP, and Ebi collaborate in a novel pathway for beta-catenin degradation linked to p53 responses. Mol Cell 2001; 7: 915-926.

73. Liu J, Stevens J, Rote CA, Yost HJ, Hu Y, Neufeld KL et al. Siah-1 mediates a novel betacatenin degradation pathway linking p53 to the adenomatous polyposis coli protein. $\mathrm{Mol}$ Cell 2001; 7: 927-936.

74. Fiucci G, Beaucourt S, Duflaut D, Lespagnol A, Stumptner-Cuvelette $P$, Geant $A$ et al. Siah- $1 \mathrm{~b}$ is a direct transcriptional target of $\mathrm{p} 53$ : identification of the functional p53 responsive element in the siah-1b promoter. Proc Natl Acad Sci USA 2004; 101 3510-3515.

75. Schubbert S, Shannon K, Bollag G. Hyperactive Ras in developmental disorders and cancer. Nat Rev Cancer 2007; 7: 295-308.

76. Carthew RW, Rubin GM. Seven in absentia, a gene required for specification of R7 cell fate in the Drosophila eye. Cell 1990; 63: 561-577.
77. Schmidt RL, Park $\mathrm{CH}$, Ahmed AU, Gundelach JH, Reed NR, Cheng S et al. Inhibition of RAS-mediated transformation and tumorigenesis by targeting the downstream E3 ubiquitin ligase seven in absentia homologue. Cancer Res 2007; 67: 11798-11810.

78. Semenza G. Signal transduction to hypoxia-inducible factor 1. Biochem Pharmacol 2002; 64: 993-998.

79. Safran M, Kaelin Jr WG. HIF hydroxylation and the mammalian oxygen-sensing pathway. J Clin Invest 2003; 111: 779-783.

80. Nakayama K, Frew IJ, Hagensen M, Skals M, Habelhah H, Bhoumik A et al. Siah2 regulates stability of prolyl-hydroxylases, controls HIF1alpha abundance, and modulates physiological responses to hypoxia. Cell 2004; 117: 941-952.

81. Aikawa Y, Nguyen LA, Isono K, Takakura N, Tagata Y, Schmitz ML et al. Roles of HIPK and HIPK2 in AML1- and p300-dependent transcription, hematopoiesis and blood vesse formation. EMBO J 2006; 25: 3955-3965. 